\title{
ACUTE PRE-B LYMPHOBLASTIC LEUKEMIA AND CONGENITAL ANOMALIES IN A CHILD WITH A DE NOVO 22q11.1q11.22 DUPLICATION
}

Vaisvilas $\mathrm{M}^{1}$, Dirse $\mathrm{V}^{2,3}$, Aleksiuniene $\mathrm{B}^{4,5}$, Tamuliene $\mathrm{I}^{6}$, Cimbalistiene L ${ }^{4,5}$, Utkus A ${ }^{1,5}$, Rascon $\mathrm{J}^{1,6}$

*Corresponding Author: Jelena Rascon, M.D., Ph.D., Center for Pediatric Oncology and Hematology, Children's Hospital, Affiliate of Vilnius University Hospital Santaros Klinikos, Santariskiu St. 4, LT 08406 Vilnius, Lithuania. Tel: +3705-2328703. Fax: +3705-272-0368. E-mail: jelena.rascon@santa.lt

\begin{abstract}
Microdeletions and microduplications are recurrent in the q11.2 region of chromosome 22. The 22q11.2 duplication syndrome is an extremely variable disorder with a phenotype ranging from severe intellectual disability, facial dysmorphism, heart defects, and urogenital abnormalities to very mild symptoms. Both benign and malignant hematological entities are rare. A male patient was diagnosed with mild facial dysmorphia, congenital heart anomalies shortly after birth and acute bowel obstruction due to malrotation of the intestine at the age of 3 years. A whole-genome single nucleotide polymorphism (SNP) array revealed a de novo $6.6 \mathrm{Mb}$ duplication in the 22q11.1q11.22 chromosomal region. A year later, the patient was diagnosed with acute pre-B lymphoblastic leukemia (pre-B ALL). Five genes, CDC45, CLTCL1, $D G C R 2, G P 1 B B$ and SEPT5, in the 22q11.1q11.22 region are potentially responsible for cell cycle division. We hypothesized that dosage imbalance of genes implicated in the rearrangement could have disrupted the balance be-
\end{abstract}

\footnotetext{
${ }^{1}$ Faculty of Medicine, Vilnius University, Vilnius, Lithuania;

${ }^{2}$ Hematology, Oncology and Transfusion Medicine Center, Vilnius University Hospital Santaros Klinikos, Vilnius, Lithuania;

${ }^{3}$ Department of Internal, Family Medicine and Oncology, Institute of Clinical Medicine of the Faculty of Medicine of Vilnius University, Vilnius, Lithuania;

${ }^{4}$ Department of Human and Medical Genetics, Institute of Biomedical Sciences of the Faculty of Medicine of Vilnius University, Vilnius, Lithuania;

${ }^{5}$ Center for Medical Genetics, Vilnius University Hospital Santaros Klinikos, Vilnius, Lithuania;

${ }^{6}$ Center for Pediatric Oncology and Hematology, Children's Hospital, Affiliate of Vilnius University Hospital Santaros Klinikos, Vilnius, Lithuania.
}

tween cell growth and differentiation and played a role in the initiation of malignancy with a hyperdiploid leukemic clone, whereas over-expression of the $T B X 1$ gene might have been responsible for congenital heart defects and mild facial dysmorphia.

Keywords: Congenital heart defect; Facial dysmorphia; Leukemia; Leukemogensis; Malrotation of the intestine; TBX1 gene; 22q11.1q11.22 duplication.

\section{INTRODUCTION}

Microdeletions and microduplications are recurrent in the q11.2 region of chromosome 22. Genomic rearrangements in this region are caused by a non allelic homologous recombination between specific low-copy repeats spanning the region. The 22q11.2 deletion is common and manifests as DiGeorge/velocardiofacial syndrome (DG/VCFS), whereas duplication in the same region is rarely reported. The 22q11.2 microduplication syndrome is an extremely variable disorder with a phenotype ranging from severe intellectual disability, facial dysmorphism, heart defects, and urogenital abnormalities to very mild symptoms [1]. Although many organs can be affected, hematological disturbances are rare. Single nucleotide polymorphism (SNP) array analysis provides a highly sensitive platform to detect large and small genomic aberrations, which could uncover rare syndomes as well as primary genomic lesions of the acute leukemia.

We report a clinical and molecular characterization of a proband with a de novo mosaic 22q11.1q11.22 duplication and acute pre-B lymphoblastic leukemia (pre-B ALL). To the best of our knowledge, only one association between duplication in the $22 \mathrm{q} 11.21$ region and pediatric pre-B ALL has been reported so far [2]. 


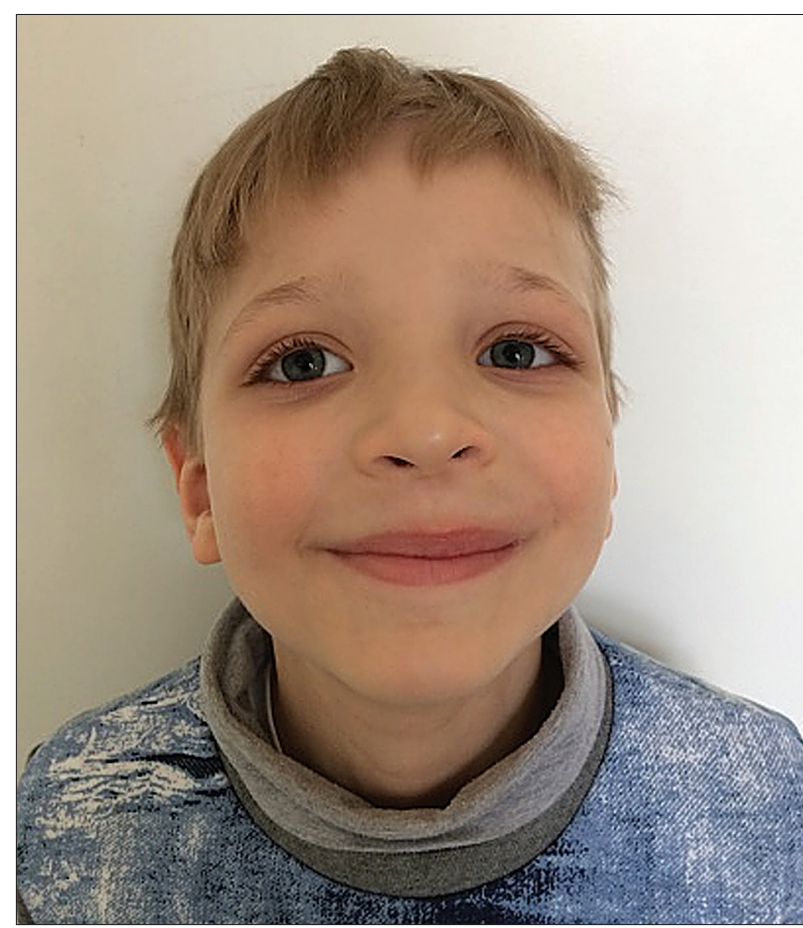

Figure 1. Dysmorphic facial features of the proband at the age of 6: a broad flat nose, down slanting palpebral fissures, hypertelorism, and mild ptosis are noted (permission of parents to publish the picture was obtained).

\section{MATERIALS AND METHODS}

Cytogenetic Analyses. Conventional chromosome analysis was carried out using G-banding techniques on stimulated peripheral blood lymphocytes according to standard laboratory protocols. A total 100 metaphases were analyzed. The karyotype was described according to the guidelines of the International System for Human Cytogenetic Nomenclature [3].

Chromosomal Microarray Analyses. The analysis was performed using the Infinium HD whole-genome genotyping assay with the HumanCytoSNP-12 BeadChip (Illumina Inc., San Diego, CA, USA) that was done according to standard protocol provided by the manufacturer. This array platform contains 299,140 SNPs distributed across the human genome with an average resolution of 31 kb. Genotypes were called by GenomeStudio Genotyping Module v2.0 (Illumina Inc.). Log R ratio and B allele frequency (BAF) values were extracted from GenomeStudio software and used in further copy number variation (CNV) analyses and breakpoint mapping with Hidden Markov Model-based QuantiSNP software (v1.1) [4]. Constitutional copy number polymorphisms were excluded based on comparison with the Database of Genomic Variants (http://projects.tcag.ca/variation).

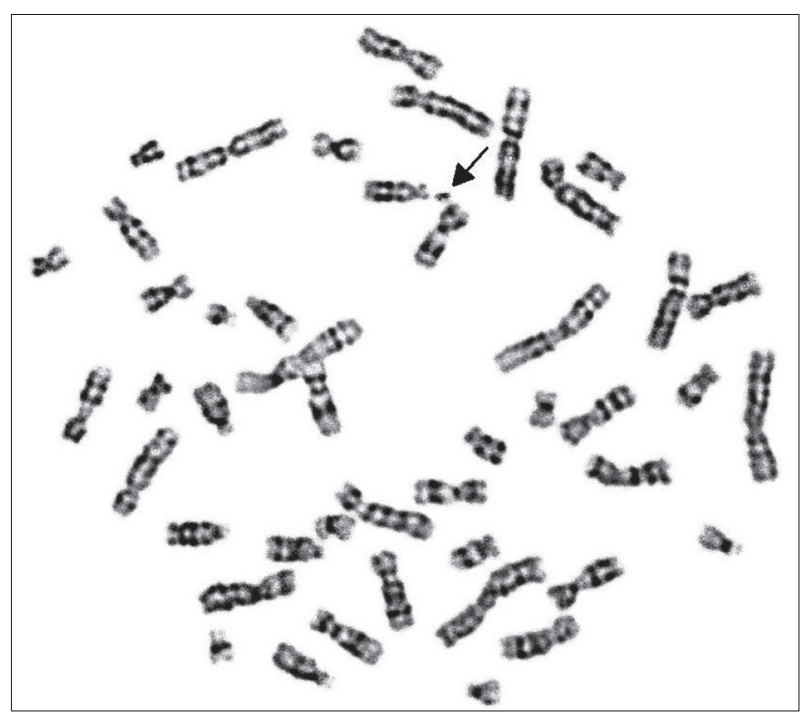

Figure 2. The marker chromosome revealed by conventional karyotyping at the age of 3 before pre-B-ALL developed is indicated by an arrow.

Case Report. A male patient, the first child of Caucasian non consanguineous parents, was born in the 40th gestational week. Weight at birth was $3900 \mathrm{~g}$ (75th percentile) and height was $59 \mathrm{~cm}$ (97th percentile). Shortly after birth, he was diagnosed with total anomalous pulmonary venous drainage, atrial septal defect and patent arterial duct that required urgent surgical care to repair this hemodynamically significant congenital heart defect. From the age of 6 months, the boy experienced episodes of periodic vomiting and abdominal cramps. Thorough investigations revealed duodenogastric reflux with multiple esophageal erosions and the intestinal malrotation.

At 3 years of age, the boy was examined by a clinical geneticist. The patient's weight, height and head circumference corresponded to the 50th percentile. His facial features appeared slightly dysmorphic with widely spaced eyes, superior placement of the eyebrows, down slanted palpebral fissures, mild ptosis, broad flat nose, opened mouth (Figure 1). His psychomotor development was borderline normal. A later review at age 5, after treatment of ALL, showed good progress and a normal development.

One year later at the age of 4 , the boy experienced an acute attack of diffuse abdominal pain and distention and was therefore rushed to the emergency department. A thorough investigation revealed an acute bowel obstruction due to malrotation of the intestines. During the next year, the patient was readmitted to the hospital several times for symptoms of infectious colitis and pancytopenia, until acute leukemia was diagnosed at the age of 4 . Thirty percent of blast cells were present in peripheral blood. A bone marrow smear revealed hypercellular bone marrow with $83.5 \%$ of blasts and a DNA 


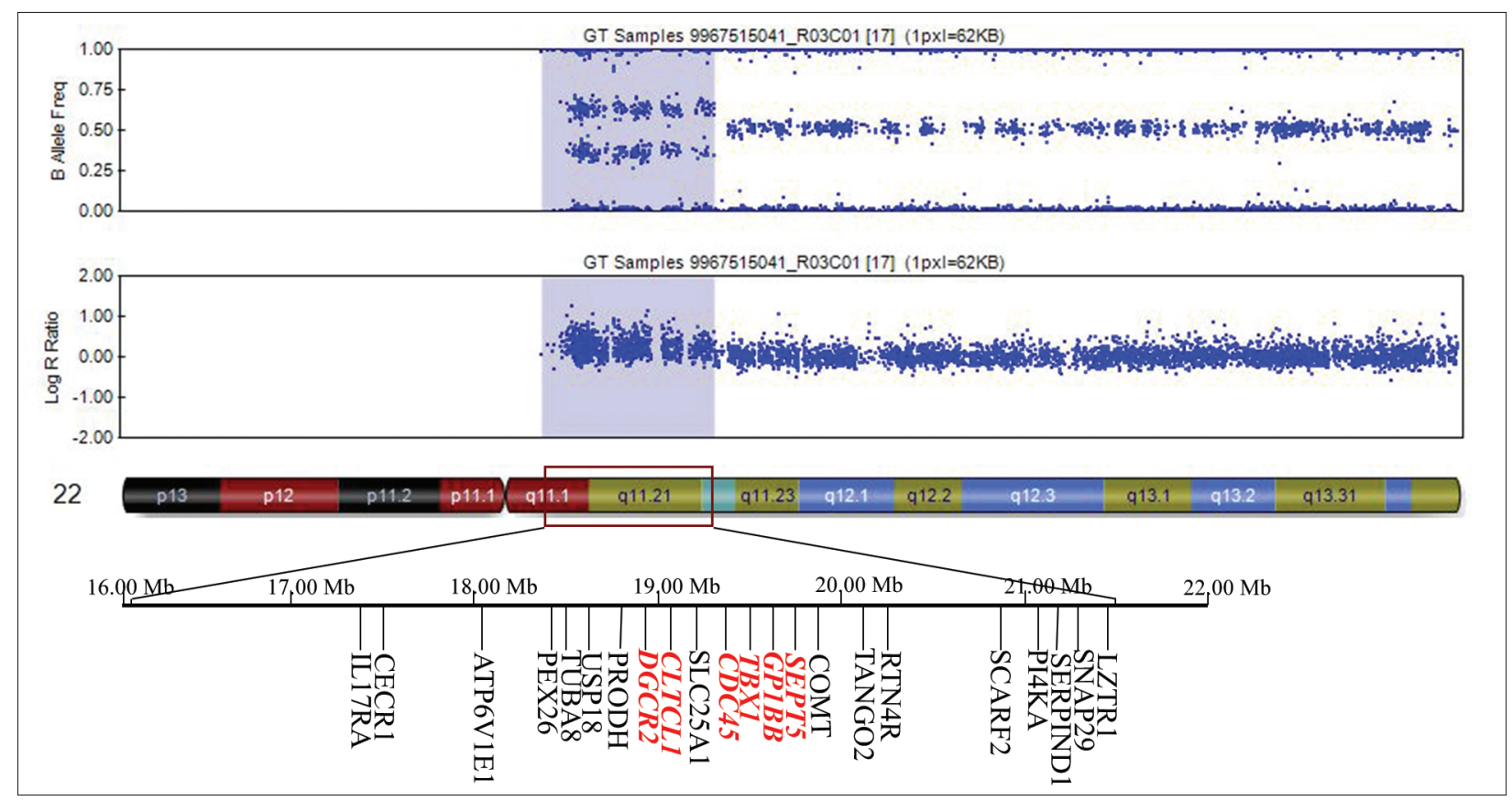

Figure 3. A de novo 6.6 Mb gain at 22q11.1-q11.22 chromosome region detected by SNP array (red arrow) at the age of 3 before pre-B-ALL developed. Genes that might play a role on oncogenesis in the area of duplication are displayed.

index of 1.2. Flow cytometry of the malignant clone in the bone marrow revealed the lymphoid origin of the blast cells with the following phenotype: CD45+, CD10+, CD38+, $\mathrm{TdT}+, \mathrm{CD} 19+, \mathrm{CD} 22+, \mathrm{cCD} 22+\mathrm{b} 1, \mathrm{cCD} 79 \mathrm{a}+, \mathrm{CD} 58+$, CD66c+, CD123+, CD13-, CD15-, CD20-, CD33-, CD34-, cCD3-, cMPO-. Hence, pre-B ALLwas diagnosed.

The patient was treated according to NOPHO ALL2008 clinical trial protocol. To date, the boy is on maintenance therapy and is in remission 2 years after diagnosis. Conventional chromosome analysis in peripheral blood cells revealed a mosaic karyotype 47,XY,+mar[85]/46, $\mathrm{XY}$ [15] (Figure 2). For further determination of the chromosomal origin of the marker chromosome, a whole-genome SNP array was carried out. The SNP array analysis revealed a $6.6 \mathrm{Mb}$ duplication in the 22q11.1q11.22 (hg[19]: chr22:16079545-22701051) region (Figure 3) and delineated the marker chromosome. Parental SNP array analysis revealed a de novo origin of the $22 \mathrm{q} 11.1 \mathrm{q} 11.22$ duplication in the proband. Duplication 22q11.1q11.22 was identified and assessed as clinically significant.

A year later for the pre-B ALL determination SNP array analysis of the leukemic bone marrow revealed the following aberrations: trisomies of chromosomes $6,11,14$, 17,18 , duplication of the $22 \mathrm{q}$ region and four signals in the $21 \mathrm{q}$ region $(R U N X 1$ gene were not included into this region). These size and the breakpoints of the $22 \mathrm{q}$ duplication was the same as in the primary sample. According to SNP array data, the final karyotype was determined as hyperdiploidic with 51 chromosomes and additional 22q and $21 \mathrm{q}$ aberrations. Conventional chromosome analysis of leukemic bone marrow was unsuccessful because of poor growth of cells.

\section{DISCUSSION}

Recent advances in the use of microarray technologies have enabled the identification of genomic rearrangements and led to further delineation of the phenotypes associated with microdeletion and microduplication syndromes. Deletions in the 22q11.22 region usually arise de novo and are relatively common. In comparison, 22q11.2 duplications are considered rare, with the estimated incidence depending on the study. Up to now more than 50 unrelated cases of 22q11.2 duplication syndrome with a high frequency of familial duplications have been reported [5].

Here we report a proband with a de novo mosaic 22q11.1q11.22 duplication. There have been numerous reports of patients with 22q11.2 deletion/duplication syndrome in the literature, but to the best of our knowledge, 22 q11.2 duplication associated with acute pre-B ALL in a child has only been reported once before [2]. Both genetic aberrations in the same region give rise to isolated or multisystemic malformations causing extremely diverse manifestations ranging from no symptoms to extreme organ malformations and neuropsychiatric syndromes. Cardiac defects are rare in duplication of the $22 \mathrm{q} 11.2$ region [6]. 
From birth, our patient had total anomalous pulmonary venous drainage, atrial septal defect and patent ductus arteriosus. Moreover, a correlation between duplications and deletions and a spectrum of neuropsychiatric disorders ranging from cognitive impairment and speech difficulties to a spectrum of psychiatric disorders such as autism or schizophrenia has been established in various studies [7]. Along with dysmorphic facial features, our patient displayed normal growth and development.

The $6.6 \mathrm{Mb}$ duplication encompasses more than 80 genes. The TBX1 gene is a member of the T-box family of transcription factors that are responsible for the formation of tissues and organs during embryonic development. The $T B X 1$ gene is expressed in the second heart field and is important for normal heart development [8]. It has been suggested that over- and/or underexpression of $T B X 1$ may affect the same developmental pathways and contribute to DG/VCFS and 22q11.2 duplication syndrome [9]. We speculated that overexpression of the $T B X 1$ gene might have been responsible for the patient's congenital heart defects and mild facial dysmorphia. Given the extremely variable clinical presentation in 22q11.2 duplication syndrome, similarities and the overlapping effects with microdeletions should be kept in mind, but always distinguished from one another.

Chang et al. [2] reported a case of a small, $0.6 \mathrm{Mb}$ duplication at the 22q11.21 region associated with pre-B ALL and cystinuria. The authors hypothesized that overexpression of the genes in the duplicated region responsible for the cell cycle and duplication might have contributed to the formation of the leukemic clone in the bone marrow. Our patient has a notably larger duplication size that encompasses a $6.6 \mathrm{Mb}$ genomic region. Both cases share a particular part of the duplication region that contains five genes potentially relevant to the regulation of cell cycle division: CDC45, CLTCL1, DGCR2, GP1BB and SEPT5. In several cases of acute leukemia the SEPT5 gene was identified as a fusion partner with $M L L$ gene. SEPT5 belongs to the human septins, a highly conserved family of guanosine triphosphate (GTP)-binding proteins. The MLLSEPTIN chimeric proteins retain the functional domains of $M L L$ (AT hook domain and methyltransferase domain) and the GTP-binding domain of the septin, suggesting an important role for the development of leukemia [10]. With regard to the SEPT5 gene, in one case with bleeding disorders, deletion of both the SEPT5 and GP1BB genes was detected [11]. It was also suggested that $C D C 45$ gene disruption could delay DNA replication and have dominant negative impact on this DNA damage response in MLLfusion acute leukemias [12]. CLTCL1, known as clathrin gene, demonstrates the presence of CLTC-ALK fusions in diffuse large B cell lymphoma (DLBCL) and extend the list of diseases associated with this genetic abnormality to include classical T-cell or null anaplastic large-cell lymphoma (ALCL), ALK+ DLBCL, and inflammatory myo-fibroblastic tumors [13]. Finally, gene expression data noticed that the DGCR2 gene was down-regulated in more than five different tumors [14]. We hypothesized that defects in any of these genes could disrupt the balance between cell growth and differentiation and play a role in the initiation of leukemia with a hyperdiploidic karyotype clone. Given the large variability of the size of these aberrations, it is possible that single gene mutations might lead to these neoplastic changes in the body rather than a larger number of amplified oncogenes, but evidence is lacking.

The risk of vincristine induced neurotoxicity is dependent on the individual activity of cytochromal enzymes, CYP3A5 in particular in patients treated for leukemia. Vincristine is metabolized more rapidly by the enzyme mentioned above. We did not perform analysis on the enzyme mentioned above, hence the true nature of enhanced toxicity is unclear for this patient. The CYP3A5 enzymes are found not only in the liver, but in the gall bladder, and the intestines as well. Since the patient had a bowel resection, it remains unclear if the toxicity is genetic, or iatro-genically induced [15].

In summary, 22q11.2 duplication syndrome is an extremely variable disorder, that can cause multiorgan system malformations or mental retardation or even present with no signs or symptoms at all. Distinguishing this syndrome from other rearrangements in the same region is very important, because of the potential association with neoplastic disease. This is the second case report of 22q11.2 duplication in association with pre-B ALL. Although only two cases have been reported so far, it seems that these patients deserve special consideration while on chemotherapy, since therapy-associated toxicity might be more prominent than usual.

Declaration of Interest. The authors report no conflicts of interest. The authors alone are responsible for the content and writing of this article.

\section{REFERENCES}

1. Wentzel C, Fernstrom M, Ohrner Y, Anneren G, Thuresson AC. Clinical variability of the 22q11.2 duplication syndrome. Eur J Med Genet. 2008; 51(6): 501-510.

2. Chang VY, Quintero-Rivera F, Baldwin EE, Woo $\mathrm{K}$, Martinez-Agosto JA, Fu C, et al. B-acute lym- 
phoblastic leukemia and cystinuria in a patient with duplication 22q11.21 detected by chromosomal microarray analysis. Pediatr Blood Cancer. 2011; 56(3): 470-473.

3. ISCN 2016. An International System for Human Cytogenomic Nomenclature. McGowan-Jordan J, Simons A, Schmid M, Eds. Basel (Switzerland): S. Karger Publishing, 2016.

4. Colella S, Yau C, Taylor JM, Mirza G, Butler H, Clouston P, et al. QuantiSNP: An objective Bayes hidden Markov model to detect and accurately map copy number variation using SNP genotyping data. Nucleic Acids Res. 2007; 35(6): 2013-2025.

5. Portnoi MF. Microduplication 22q11.2: A new chromosomal syndrome. Eur J Med Genet. 2009; 52(2-3): 88-93.

6. Rosa RF, Zen PR, Ricachinevsky CP, Pilla CB, Pereira VL, Roman T, et al. 22q11.2 duplication and congenital heart defects. Arq Bras Cardiol. 2009; 93(4): e55-e57.

7. Schmock H, Vangkilde A, Larsen KM, Fischer E, Birknow MR, Jepsen JR, et al. The Danish 22q11 research initiative. BMC Psychiatry. 2015; 15: 220.

8. Bruneau BG. The developmental genetics of congenital heart disease. Nature. 2008; 451(7181): 943-948.

9. Ou Z, Berg JS, Yonath H, Enciso VB, Miller DT, Picker J, et al. Microduplications of 22q11.2 are frequently inherited and are associated with variable phenotypes. Genet Med. 2008; 10(4): 267-277.
10. Launay E, Henry C, Meyer C, Chappe C, Taque S, Boulland ML, et al. MLL-SEPT5 fusion transcript in infant acute myeloid leukemia with $\mathrm{t}(11 ; 22)$ (q23;q11). Leuk Lymphoma. 2014; 55(3): 662-667.

11. Bartsch I, Sandrock K, Lanza F, Nurden P, Hainmann I, Pavlova A, et al. Deletion of human GP1BB and SEPT5 is associated with Bernard-Soulier syndrome, platelet secretion defect, polymicrogyria, and developmental delay. Thromb Haemost. 2011; 106(3): 475-483.

12. Schnerch D, Yalcintepe J, Schmidts A, Becker H, Follo M, Engelhardt M, et al. Cell cycle control in acute myeloid leukemia. Am J Cancer Res. 2012; 2(5): 508-528.

13. Gascoyne RD, Lamant L, Martin-Subero JI, Lestou VS, Harris NL, Muller-Hermelink HK, et al. ALKpositive diffuse large B-cell lymphoma is associated with Clathrin-ALK rearrangements: Report of 6 cases. Blood. 2003; 102(7): 2568-2573.

14. Chen S, Zhu B, Yu L. In silico comparison of gene expression levels in ten human tumor types reveals candidate genes associated with carcinogenesis. Cytogenet Genome Res. 2006; 112(1-2): 53-59.

15. Egbelakin A, Ferguson MJ, MacGill EA, Lehmann AS, Topletz AR, Quinney SK, et al. Increased risk of vin-cristine neurotoxicity associated with low CYP3A5 expression genotype in children with acute lymphoblastic leukemia. Pediatr Blood Cancer. 2011; 56(3): 361-367. 